\title{
SIRT6 is upregulated and associated with cancer aggressiveness in papillary thyroid cancer via BRAF/ERK/Mcl-1 pathway
}

\author{
NING QU ${ }^{1 *}$, JIA-QIAN HU $^{1 *}$, LIANG LIU ${ }^{2}$, TING-TING ZHANG ${ }^{1}$, \\ GUO-HUA SUN ${ }^{1}$, RONG-LIANG SHI ${ }^{1}$ and QING-HAI JI ${ }^{1}$
}

\author{
Departments of ${ }^{1}$ Head and Neck Surgery, and ${ }^{2}$ Radiology, Fudan University Shanghai Cancer Center; \\ Department of Oncology, Shanghai Medical College, Fudan University, Shanghai 200032, P.R. China
}

Received January 10, 2017; Accepted February 15, 2017

DOI: $10.3892 /$ ijo.2017.3951

\begin{abstract}
Sirtuin 6 (SIRT6) is a member of the SIRT family $\mathrm{NAD}^{+}$-dependent deacetylases reported to function in controlling organism homeostasis, lifespan, and diseases. This study investigated the role of SIRT6 in papillary thyroid cancer (PTC). Data of 391 PTC patients was extracted from The Cancer Genome Atlas database to investigate the expression of SIRTs (SIRT1-7) and their relationship with clinicopathological parameters. Additional 45 pairs of PTC tumor tissues and corresponding non-tumor tissues were studied using microarray analysis for SIRT6 expression. Surgically resected, pathologically diagnosed tissues from 130 in-house PTC patients were used for confirmation of SIRT6 expression. SIRT6 silenced K1 and TPC-1 cells were generated to explore the influence of SIRT6 on cancer cell aggressiveness in vitro. SIRT6 mRNA and protein levels were upregulated in PTC tumor tissues and its overexpression was an independent biomarker for nodal metastasis (odds ratio $=1.794,95 \%$ confidence interval: $1.256-1.920, \mathrm{p}=0.012$ ). SIRT6 expression was related to poor recurrence-free survival, however, not significantly. Silencing SIRT6 downregulated PTC cell aggressiveness in vitro by suppressing ERK and Mcl-1. In conclusion, these results suggest that SIRT6 enhances cell aggressiveness in PTC via BRAF/ERK/Mcl-1 pathway, and thus may be a promising target in the treatment of the disease.
\end{abstract}

\section{Introduction}

The incidence of papillary thyroid cancer (PTC) has been rising dramatically for the past three decades $(1,2)$. It was the

Correspondence to: Professor Qing-Hai Ji, Department of Head and Neck Surgery, Fudan University Shanghai Cancer Center; Department of Oncology, Shanghai Medical College, Fudan University, Shanghai 200032, P.R. China

E-mail: jiqinghai@shca.org.cn

*Contributed equally

Key words: SIRT6, papillary thyroid cancer, BRAF, ERK, Mcl-1 fifth most commonly diagnosed type of cancer among women in United States in 2015 (3). The prognosis of PTC patients is known to be excellent, with a 10 -year survival rate exceeding $90 \%(4,5)$. Nevertheless, up to $10 \%$ of patients would eventually die of the disease and an even greater proportion would face recurrence $(6,7)$. Therefore, avoiding recurrence and early diagnosis of recurrent disease is of great importance in the long term management of PTC. Efforts have been made in identifying various clinicopathological as well as biological predictors for PTC. Using these predictors, risk-group stratification or staging systems can thus be established and be applied to differentiate PTC prognosis. The ultimate purpose is to treat PTC patients individually and hopefully, lower the recurrence rate of the disease. Undisputedly, in order to reach this goal, a better understanding of the PTC pathogenesis and progression is needed.

Sirtuins (SIRTs) are a class of $\mathrm{NAD}^{+}$-dependent protein deacetylases involved in stress resistance and metabolic homeostasis. There are 7 members in the family: SIRT1-SIRT7. Many of these proteins have been reported to regulate important pathways and are responsible for vital biological process (8-10). SIRT6, like the other members in the family, also exhibits many pivotal functions and multiple enzymatic activities (11-13). It has been reported to be in connection with a number of human diseases, including cancer (14-20). However, whether SIRT genes are involved in PTC has not been elucidated yet.

This study aimed to investigate the roles of SIRTs in PTC. We analyzed all SIRT family genes in 391 PTC cases from The Cancer Genome Atlas (TCGA) cohort and an additional 45 PTC cases from Microarray data. Tissues from 130 in-house surgically treated, pathologically confirmed PTC patients were used for verification of SIRT6 expression. We also generated SIRT6 silenced K1 and TPC-1 cells to explore the influence of SIRT6 on cancer cell aggressiveness.

\section{Materials and methods}

Patients and tissue samples. This study acquired Institutional Review Board approval from Fudan University Shanghai Cancer Center (Shanghai, China). Written informed consent was obtained from all subjects. The methods were carried out in accordance with the approved guidelines. 
Pathologically confirmed thyroid cancer tissues, were obtained from 130 patients who received thyroidectomy between 2012 and 2015 at the Department of Head and Neck Surgery, Fudan University Shanghai Cancer Center. For the TCGA cohort, SIRT gene expression and clinical data of TCGA database are available from the website of Cancer Genomics Browser of University of California Santa Cruz (UCSC) (https://genome-cancer.ucsc.edu/). A total number of 7 members of the SIRT family are included in the database. Patients who had PTC as the only malignancy or the first of multiple malignancies and received surgical therapy were included. Other inclusion criteria were: patients with no pre-treatment, with sufficient description of primary tumors and intact recurrence-free survival (RFS)/overall survival (OS) information. Follow-up was completed on December 21, 2014. The patients were excluded if with: insufficient data or unknown clinicopathologic profile, undetermined histology, other types of thyroid cancer (follicular thyroid cancer, medullary thyroid cancer and anaplastic thyroid cancer), or secondary tumor. The following variables were used for the analysis: gender, age at diagnosis, maximal tumor size, bilateral lesions, multifocal lesions, and lymph node status. Patients were staged using the latest 7th AJCC TNM staging system according to the final pathology reports. The primary tumor and nodal status were assessed in (or by) the final pathology. Demographic and clinicopathological characteristics of enrolled patients were obtained from electronic records and summarized in Table I.

Selection of cut-off value. X-tile software (version 3.6.1, Yale University School of Medicine, Newhaven, CT, USA) was used to divide the TCGA cohort according to SIRT6 expression into 3 subsets: low, middle and high. X-tile plots were generated for assessment of SIRT6 expression and RFS, optimization of cut-points and correction by the use of minimum p statistics.

Microarray data analysis. Microarray data discussed in this study have been deposited in the Gene Expression Omnibus (GEO) of U.S. National Center for Biotechnology Information (NCBI; Bethesda, MD, USA; http://www.ncbi.nlm.nih. gov/geo). The results of the microarray experiment are accessible through GEO series accession number GSE33630.

We hybridized 45 PTC tissues and the corresponding para tumor tissues onto Affymetrix U133 Plus 2.0 arrays. Paired RNA samples of PTCs and non-tumor thyroid tissues were obtained from Ukraine via the Chernobyl Tissue Bank (www.chernobyltissuebank.com). Diagnoses were confirmed by the members of the International Pathology Panel of the Chernobyl Tissue Bank.

Immunohistochemistry (IHC) staining and scoring. IHC staining was carried out according to the manufacturer's instruction. Briefly, formalin-fixed and paraffin-embedded tissue sections were deparaffinized in xylene and hydrated with decreasing concentrations of ethanol (100, 95, 80 and 75\%). The slices were then soaked in $10 \%$ BSA to inhibit endogenous peroxidase activity and incubated with SIRT6 rabbit polyclonal antibody (working dilution 1:50; Proteintech Group Inc., Chicago, IL, USA) at $4{ }^{\circ} \mathrm{C}$ overnight. A horseradish peroxidase-conjugated rabbit secondary antibody was added for $60 \mathrm{~min}$ at room temperature; then, 3,3'-diaminobenzidine
Table I. Clinical characteristics of patients with papillary cancer in The Cancer Genome Atlas cohort.

TCGA

Variables

No. $(\%)$, total $n=391$

Gender
Male
Female
Age, years
Mean age
$\leq 45$
$>45$

$108(27.6)$

$283(72.4)$

$47.0 \pm 15.8$

$192(49.1)$

199 (50.9)

First degree relative history

No

$371(94.9)$

Yes

$20(5.1)$

Histological type

Classical/usual

275 (70.3)

Follicular

85 (21.8)

Tall cell

31 (7.9)

Primary tumor size

Mean size

$<2 \mathrm{~cm}$

$246(62.9)$

2-4 cm

$136(34.8)$

$9(2.3)$

Primary tumor focus

Unifocal

$226(57.8)$

Multifocal

165 (42.2)

Residual tumor

R0

307 (78.5)

44 (11.2)

$40(10.3)$

R2

$331(84.7)$

$60(15.3)$

Positive

105 (26.9)

139 (35.5)

$129(33.0)$

18 (4.6)

Pathologic N stage

NO

217 (55.5)

N1

$174(44.5)$

Pathologic M stage

M0

383 (98.0)

M1

8 (2.0)

Mean age and mean size are presented as mean \pm standard deviation.

(DAB) development (DAB Substrate Chromogen System; Dako, Glostrup, Denmark) and hematoxylin staining were performed according to standard protocols. Slides were fixed and images were obtained through an Olympus IX71 inverted 
microscope with a DP2-BSW Olympus image acquisition software system. The sections were read separately by two experienced pathologists blinded to the clinicopathologic data. The sections were scored on the basis of the extent of staining ( 0 , no staining; $1, \leq 10 \% ; 2,10-50 \%$; and $3,>50 \%$ ) and the staining intensity ( 0 , negative; 1 , weak; 2 , moderate; and 3, strong). These scores were multiplied to calculate an immunoreactivity score (IS) for each case.

Cell lines and cell culture. Two human PTC cell lines (TPC-1 and K1) were used in this study. TPC-1 cell line was kindly provided by Professor Haixia Guan from China Medical University (Shenyang, China). K1 cell line was purchased from University of Colorado Cancer Center Cell Bank. All cells were cultured in RPMI-1640 medium supplemented with $10 \%$ FBS (Invitrogen, Carlsbad, CA, USA) at $37^{\circ} \mathrm{C}$ with $5 \% \mathrm{CO}_{2}$.

shRNA plasmid and transfection. Two separate sequences (GCAGTCTTCCAGTGTGGTGTT and GCTGGGTAC ATCGCTGCAGAT) of shRNA targeting SIRT6 gene were designed and cloned into a pLKO.1 TRC (Addgene plasmid 10878) cloning vector. Scramble RNA (Addgene plasmid 1864) was used as control vector. Knockdown of SIRT6 in TPC-1 and K1 cells was accomplished by lentiviral infection. All these plasmids were transfected into PTC cells using Lipofectamine ${ }^{\circledR} 2000$ under the instruction of the product manual (Invitrogen). The knockout of SIRT6 was confirmed by western blotting.

$R N A$ extraction, reverse transcription and quantitative $P C R$ ( $q P C R)$. Total RNA was extracted from 130 pairs of surgically treated, pathologically diagnosed PTC tissues and cultured PTC cells using TRIzol Reagent (Invitrogen). cDNA was then obtained through reverse transcription using $1 \mu \mathrm{g}$ of total RNA with a PrimeScript ${ }^{\mathrm{TM}}$ RT reagent kit (Takara Bio, Inc., Otsu, Japan). The SIRT6 expression status was assessed by Real-time PCR, which was carried out in triplicate by a SYBR Premix Ex Taq ${ }^{\mathrm{TM}}$ kit (Takara Bio) and ABI 7900HT Real-Time PCR system (Applied Biosystems Life Technologies, Foster City, CA, USA). The SIRT6 primers used in qPCR are as follows: Forward: 5'-ACTCGCCGATGAGGCCAGCAGGAA-3'; reverse: 5'-ATGCGGAGGTCAGCATGGCGGTCGT-3'. $\beta$-actin was used as an internal control for mRNA assays. The comparative cycle threshold values $\left(2^{-\Delta \Delta \mathrm{Ct}}\right)$ were adopted to analyze the final results.

Colony formation assay. TPC-1 and K1 cells were digested and were seeded at 200 cells per well in a 6-well plate. After incubation for 5 days, colonies were fixed in methanol and stained with $0.1 \%$ crystal violet solution for $30 \mathrm{~min}$. The cells were then washed three times with PBS and clones were counted under an inverted microscope. Clones were defined as colonies of $\geq 50$ cells. Three different fields were counted for each cell culture and the average was calculated.

ATPLite luminescence assay. PTC cell proliferation was determined using the ATPLite luminescent assay (PerkinElmer, Inc., Waltham, MA, USA) according to the manufacturer's instructions. Cells were incubated in 96-well plates for $120 \mathrm{~h}$ before testing.

Western blot analysis. Cell lysates were obtained from $1 \times 10^{6}$ cells with RIPA lysis buffer (containing $50 \mathrm{mM}$ Tris-HCl, pH 7.4, 150 mM NaCl, 0.1\% SDS, 1 mM EDTA, $1 \%$ Triton X-100, $1 \mathrm{mM}$ PMSF, and $1 \mathrm{mM}$ Protease Inhibitor Cocktail). Approximately $20 \mu \mathrm{g}$ protein was extracted from each sample and separated by $10 \%$ SDS-PAGE gels. Proteins were then transferred to nitrocellulose membranes $(0.45 \mathrm{~mm}$; Solarbio, Beijing, China). After blocking the membrane with $5 \%$ non-fat milk, it was immunoblotted with primary antibodies: SIRT6 rabbit polyclonal antibody (Proteintech Group Inc.) and GAPDH (1:5,000; Abcam, Cambridge, MA, USA). The bands were visualized with 1-step ${ }^{\mathrm{TM}}$ NBT/BCIP reagents (Thermo Fisher Scientific, Rockford, IL, USA) and developed by Alpha Imager (Alpha Innotech, San Leandro, CA, USA).

In vitro migration and invasion assays. Cell migration and invasion were analyzed by a transwell plate (24-well insert, $8 \mu \mathrm{m}$ pore size; BD Biosciences, Bedford, MA, USA). The filters (Corning Inc., Acton, MA, USA) were coated with (invasion) or without (migration) $40 \mu \mathrm{l}$ Matrigel (1:8 dilution; BD Biosciences). SIRT6-silenced TPC-1 and SIRT6-silenced $\mathrm{K} 1$ cells were used in these assays. For migration assays, $3 \times 10^{4}$ cells were suspended in $100 \mu \mathrm{l}$ serum-free medium and seeded into the Matrigel-uncoated upper chambers. A total of $600 \mu \mathrm{l}$ of medium containing $10 \%$ serum was added to the lower chamber as a chemoattractant. After incubation at $37^{\circ} \mathrm{C}$ for $72 \mathrm{~h}$, non-invading cells were wiped off the upper surface with a cotton swab. The membranes were fixed with $4 \%$ formaldehyde for $20 \mathrm{~min}$ and stained with $0.1 \%$ crystal violet at room temperature. For invasion assays, $1 \times 10^{5}$ cells were used with an incubation time of $48 \mathrm{~h}$. The rest of the protocol was identical to that described above. The cells were counted and photographed under an inverted microscope over 10 different fields of each triplicate filter.

Cell cycle distribution. To evaluate the effect of silenced SIRT6 on PTC cell cycle progression, cell cycle distribution was detected by flow cytometry in TPC-1 and K1 cells after lentivirus transduction. Briefly, $1.0 \times 10^{6}$ cells of each sample were harvested, fixed in $70 \%$ ethanol, and stored at $4^{\circ} \mathrm{C}$ overnight. Cells were then stained with $\mathrm{NaCl} / \mathrm{Pi}$ staining solution (50 $\mu \mathrm{g} / \mathrm{ml} \mathrm{PI} \mathrm{and} 100 \mu \mathrm{g} / \mathrm{ml}$ RNase A) for $1 \mathrm{~h}$ in the dark at room temperature followed by flow cytometry (FACSCalibur; BD Biosciences, San Jose, CA, USA) analysis. The fractions of the cells in G1, S, and G2/phases were calculated with dedicated software (BD Biosciences).

Statistical analysis. All statistical analysis was performed using SPSS software (version 19.0, IBM Corp., Armonk, NY, USA). Independent t-tests (for continuous variables) and Pearson's $\chi^{2}$ tests (for categorical variables) were used. Continuous variables were categorized using certain cut-offs. The age of 45 was used as the cut-off point to divide all patients into two groups: younger patients ( $\leq 45$ years old) and older patients ( $>45$ years old). The odds ratio (OR) for relationships between clinicopathological factors, SIRT gene expression and regional node metastasis were calculated using 
Table II. Univariate and multivariate logistic regression analysis of SIRT gene expression and regional node metastasis for patients with papillary thyroid cancer in the TCGA cohort.

\begin{tabular}{|c|c|c|c|c|}
\hline \multirow[b]{2}{*}{ Variables } & \multicolumn{2}{|c|}{ Univariate analysis } & \multicolumn{2}{|c|}{ Multivariate analysis } \\
\hline & OR $(95 \% \mathrm{CI})$ & P-value & OR $(95 \% \mathrm{CI})$ & P-value \\
\hline Gender (female vs. male) & $0.745(0.517-1.381)$ & 0.402 & $0.664(0.367-1.201)$ & 0.176 \\
\hline Age ( $>45$ vs. $\leq 45$ years) & $0.703(0.452-1.094)$ & 0.219 & $0.597(0.350-1.018)$ & 0.058 \\
\hline First degree relative history & $0.651(0.349-2.718)$ & 0.719 & $0.992(0.311-3.165)$ & 0.989 \\
\hline Histological type & $0.510(0.353-0.739)$ & 0.001 & $0.540(0.416-0.984)$ & 0.042 \\
\hline Primary tumor focus & $1.226(0.789-1.904)$ & 0.365 & $1.064(0.634-1.786)$ & 0.814 \\
\hline Hashimoto's thyroiditis & $1.393(0.747-2.525)$ & 0.208 & $2.172(0.585-4.348)$ & 0.694 \\
\hline Pathologic T stage & $2.020(1.340-3.650)$ & 0.001 & $1.777(1.279-2.468)$ & 0.001 \\
\hline Pathologic M stage & $0.499(0.051-2.207)$ & 0.324 & $0.398(0.063-2.503)$ & 0.326 \\
\hline SIRT1 & $0.731(0.418-0.881)$ & 0.004 & $0.917(0.686-1.507)$ & 0.065 \\
\hline SIRT2 & $0.937(0.713-1.232)$ & 0.642 & $0.854(0.639-1.142)$ & 0.287 \\
\hline SIRT3 & $0.714(0.313-1.627)$ & 0.422 & $0.612(0.252-1.485)$ & 0.277 \\
\hline SIRT4 & $1.031(0.501-2.122)$ & 0.933 & $0.995(0.470-2.106)$ & 0.989 \\
\hline SIRT5 & $1.019(0.554-1.876)$ & 0.951 & $1.001(0.513-1.952)$ & 0.999 \\
\hline SIRT6 & $1.659(1.120-2.812)$ & 0.007 & $1.794(1.256-1.920)$ & 0.012 \\
\hline SIRT7 & $0.976(0.824-1.158)$ & 0.784 & $1.005(0.837-1.208)$ & 0.956 \\
\hline
\end{tabular}

SIRT, sirtuin; TCGA, The Cancer Genome Atlas; CI, confidence interval; OR, odds ratio.

binary logistic regression. For the survival analysis, patients who were alive and did not relapse were censored at the date of their last follow-up visit. Neck or distant RFS was defined as the time between the date of initial surgery and the first adverse event or death. OS was defined as the time between the date of initial surgery and death (all causes or cancer-specific). Survival rates were estimated by the Kaplan-Meier method. The hazard ratio (HR) for relationships between each variable and recurrence were calculated using binary Cox regression model. $\mathrm{P}<0.05$ was considered to indicate a statistically significant difference.

\section{Results}

Patient characteristics and main outcomes in follow-up (TCGA cohort). We identified 391 eligible patients with PTC in TCGA cohort. There were 108 (27.6\%) males and $283(72.4 \%)$ females. The mean age was 47.0 years (range from 15 to 89 years). Of these patients, 275 (70.3\%) had been confirmed as classical/usual papillary variant histologically, while follicular and tall cell variants were found in $85(21.8 \%)$ and $31(7.9 \%)$ patients, respectively. Combined with the size and extrathyroidal-extensional status of primary tumor, 105 cases $(26.9 \%)$ were classified as T1 stage, 139 (35.5\%) were T2 stage, 129 (33.0\%) were T3 stage, and only $18(1.6 \%)$ were T4 stage. According to the pathology, $174(44.5 \%)$ patients had lymph node metastasis involving either the central compartment (N1a) or both central and lateral compartment (N1b). Using the latest $7^{\text {th }}$ AJCC TNM staging system, there were 220 patients $(56.3 \%)$ with PTC stage I, $43(11.0 \%)$ with stage II, $86(22.0 \%)$ with stage III, and $42(10.7 \%)$ with stage IV. The other clinicopathological characteristics, such as first degree relative history, the primary tumor focus, the coexistent Hashimoto's thyroiditis, and the surgical completeness (residual tumor) of all patients are summarized in Table I.

The median follow-up duration was 574 days (range 1-4,780 months). During this time, 9 patients $(2.3 \%)$ died from a specific cancer cause. Disease recurrence occurred in 26 patients $(6.6 \%)$, including neck and distant recurrence. The OS rates were $92.0 \%$ at 1,854 days (approximately 5 years) and $88.0 \%$ at 2,973 days (approximately 8 years) in this cohort. On the other hand, the RFS rates were $86.1 \%$ at 1,785 days (approximately 5 years) and $83.9 \%$ at 2,093 days (approximately 6 years) in this cohort.

Influence of clinicopathological characteristics and SIRT genes expression on lymph node metastasis and RFS. The Pearson's $\chi^{2}$ tests was used to analyze the influences of clinicopathologic variables and genes expression on regional node metastasis. The results showed that the risk of nodal metastasis was significantly increased by classical papillary variant (compared to follicular or tall cell variant), advanced T stage, and higher expression of SIRT6. Instead of limiting the multivariate analysis to the significant terms from the univariate analysis, we included all variables because these factors had been previously demonstrated to be important in predicting disease recurrence in adult PTC patients. The results are presented in Table II. The risk of nodal metastasis still increased with the independent variables identified by univariate analysis. In particular, the expressions of SIRT6 $[\mathrm{OR}=1.794,95 \%$ confidence interval (CI): 1.256-1.920, $\mathrm{p}=0.012]$ was an independent biomarker predictor of nodal metastasis (Table II). 
Table III. Univariate and multivariate Cox proportional hazards analysis of SIRT gene expression and recurrence free survival for patients with papillary thyroid cancer in the TCGA cohort.

\begin{tabular}{|c|c|c|c|c|}
\hline \multirow[b]{2}{*}{ Variables } & \multicolumn{2}{|c|}{ Univariate analysis } & \multicolumn{2}{|c|}{ Multivariate analysis } \\
\hline & HR $(95 \% \mathrm{CI})$ & P-value & HR $(95 \% \mathrm{CI})$ & P-value \\
\hline Gender (female vs. male) & $0.427(0.188-0.869)$ & 0.032 & $0.684(0.190-2.457)$ & 0.760 \\
\hline Age ( $>45$ vs. $\leq 45$ years) & $1.587(0.669-4.693)$ & 0.304 & $1.271(0.305-7.296)$ & 0.742 \\
\hline First degree relative history & $0.673(0.083-5.439)$ & 0.710 & $0.770(0.041-14.334)$ & 0.861 \\
\hline Histological type & $1.189(0.607-2.292)$ & 0.666 & $1.095(0.392-4.057)$ & 0.862 \\
\hline Primary tumor focus & $0.424(0.194-1.037)$ & 0.068 & $0.303(0.067-1.370)$ & 0.121 \\
\hline Residual tumor & $1.086(0.658-1.727)$ & 0.795 & $2.489(0.760-3.917)$ & 0.246 \\
\hline Hashimoto's thyroiditis & $2.040(0.624-4.747)$ & 0.177 & $1.340(0.304-5.909)$ & 0.699 \\
\hline Pathologic T stage & $2.044(0.652-3.671)$ & 0.858 & $0.912(0.408-2.082)$ & 0.845 \\
\hline Pathologic N stage & $1.871(0.801-4.368)$ & 0.148 & $3.348(0.930-12.048)$ & 0.064 \\
\hline Pathologic M stage & $0.989(0.120-8.132)$ & 0.992 & $0.108(0.005-2.513)$ & 0.165 \\
\hline SIRT1 & $0.361(0.130-1.005)$ & 0.051 & $0.339(0.114-1.006)$ & 0.051 \\
\hline SIRT2 & $0.613(0.295-1.274)$ & 0.190 & $0.596(0.268-1.323)$ & 0.203 \\
\hline SIRT3 & $1.935(0.967-3.875)$ & 0.062 & $1.522(0.658-3.519)$ & 0.326 \\
\hline SIRT4 & $1.137(0.484-2.674)$ & 0.768 & $1.881(0.592-5.980)$ & 0.284 \\
\hline SIRT5 & $1.988(0.460-8.587)$ & 0.358 & $2.588(0.480-13.961)$ & 0.269 \\
\hline SIRT6 & $1.400(1.183-2.873)$ & 0.021 & $1.466(1.195-1.913)$ & 0.086 \\
\hline SIRT7 & $1.689(0.742-3.842)$ & 0.212 & $1.170(0.414-3.305)$ & 0.767 \\
\hline
\end{tabular}

SIRT, sirtuin; TCGA, The Cancer Genome Atlas; CI, confidence interval; HR, hazard ratio.

A

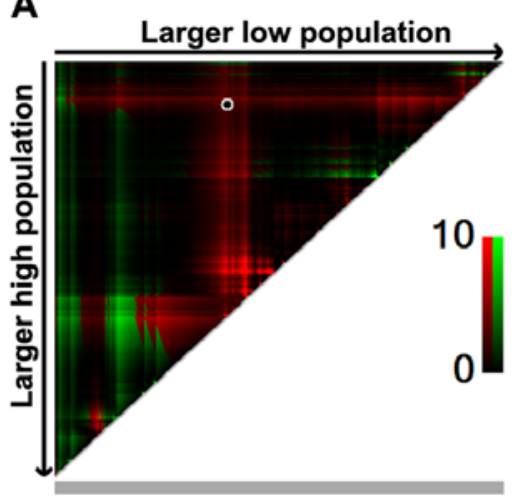

B

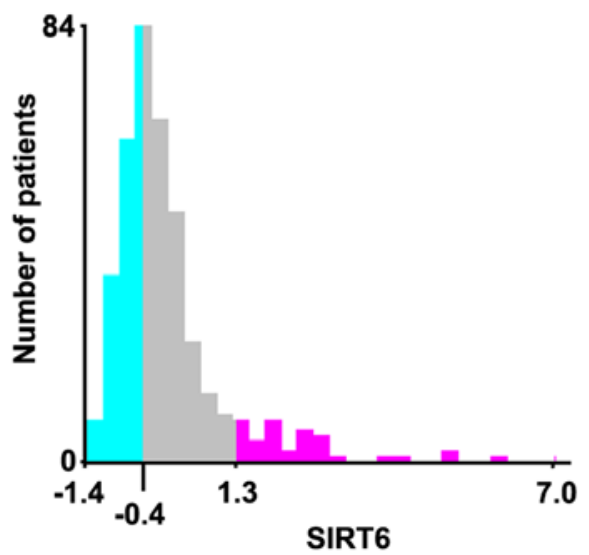

C

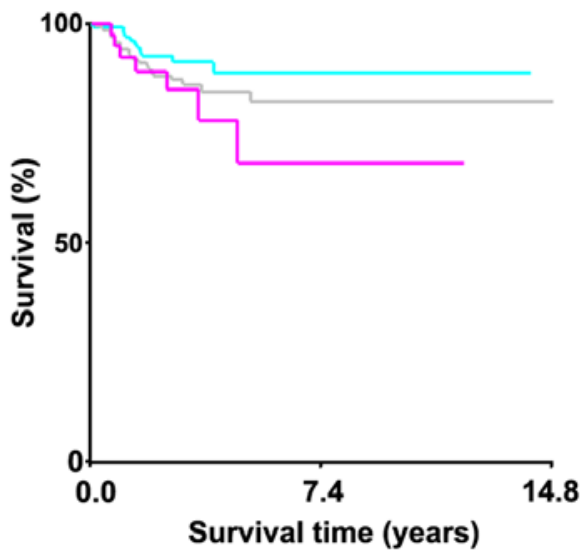

Figure 1. X-tile plots of SIRT6 gene expression on TCGA cohort. The TCGA cohort was used for X-tile analysis. (A) The cut-points and $\chi^{2}$ log-rank value created when the patients were divided into 3 subsets. (B) A histogram showing the entire cohort and cut-points (low-middle, -0.4; middle-high, 1.3). (C) A Kaplan-Meier plot showing the recurrence-free survival of these 3 subsets. SIRT6, sirtuin 6.

The Kaplan-Meier method and log-rank test showed that higher expression of SIRT6 was an independent predictor for disease recurrence, while none of the clinicopathological characteristics was significantly associated (Table III). However, multivariate analysis after adjustment for all the potential prognostic factors demonstrated that the expression of SIRT6 (HR $=1.466,95 \%$ CI: 1.195-1.913, $\mathrm{p}=0.086)$ lacked significance as an independent predictor of RFS (Table III).

To further explore the relationship between SIRT6 expression and RFS, we used X-tile software to calculate the cutoff values on TCGA cohort. According to their SIRT6 expression, we divided the cohort into three subpopulations: low, middle and high. X-tile plots were generated accordingly and the maximum of $\chi^{2} \log$-rank values was produced (Fig. 1). The low-middle cut-point was -0.4 and the middle-high cut-point was 1.3 , individually. The three subsets displayed a significant difference on Kaplan-Meier plot, which showed that the patients with the highest expression of SIRT6 had the worst RFS and those who possessed lowest expression of the gene had the best RFS. 
A

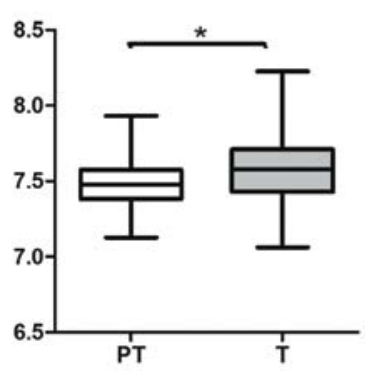

C

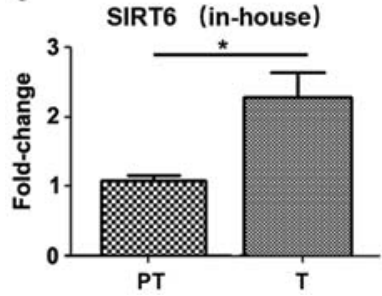

B
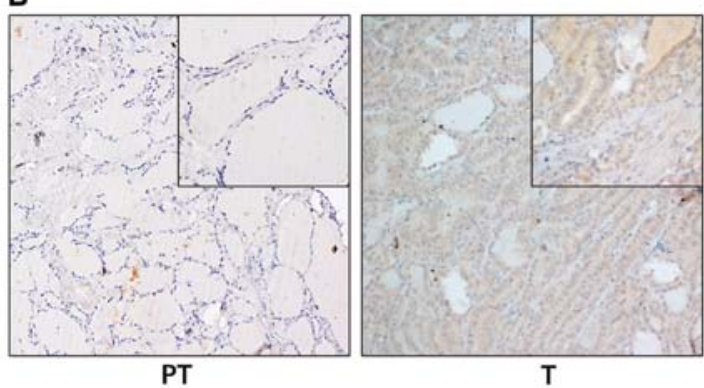

D

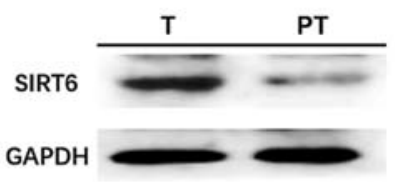

Figure 2. SIRT6 expression was upregulated in PTC tissues. (A) Microarray data (GSE33630) showed SIRT6 expression was significantly higher in PTC tumor tissues. (B) IHC staining of SIRT6 expression in formalin-fixed, paraffin-embedded PTC and corresponding non-tumor thyroid tissues (original magnification, x200). SIRT6 was expressed strongly positive in PTC tissues and almost negative in normal thyroid regions. (C) In-house surgically treated and pathologically diagnosed PTC patients showed elevated SIRT expression in tumor tissues. (D) Western blot analysis of SIRT6 in PTC. SIRT6, sirtuin 6; PTC, papillary thyroid cancer; IHC, immunohistochemistry.

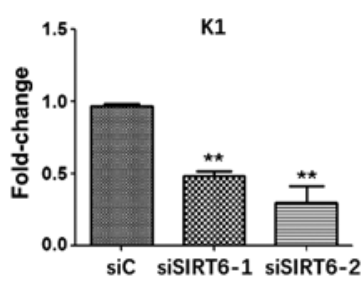

C
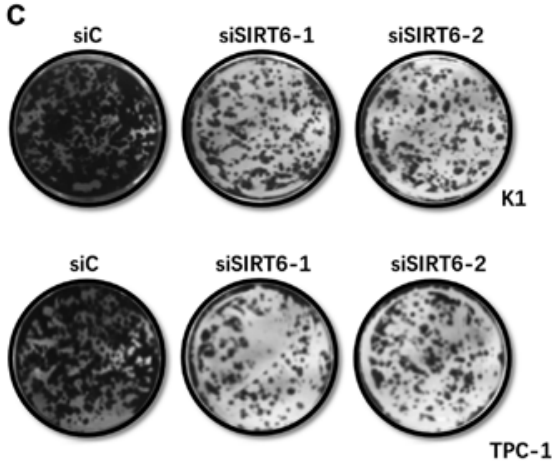

D

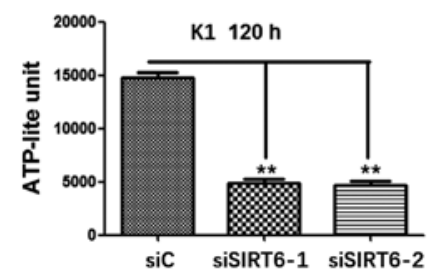

B
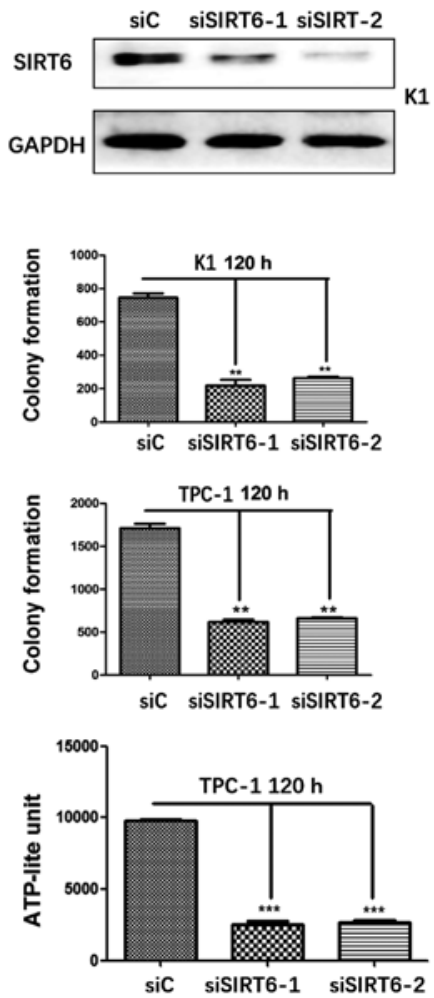

Figure 3. Silencing SITR6 weakened PTC cell proliferation in vitro. SIRT6-silenced TPC-1 and SIRT6-silenced K1 cells were generated. (A) SIRT6 expression in normal K1 cells, SIRT6-1 silenced and SIRT6-2 silenced K1 cells were analyzed by qPCR. The results were normalized to $\beta$-actin mRNA expression. (B) SIRT6 expression confirmed by western blotting. (C) Silenced SIRT6 decreased clone formation capability of K1 cells and TPC-1 cells. (D) Silencing SIRT6 inhibits cell viability reflected by ATPlite Luminescence Assay. ( $\left.{ }^{*} \mathrm{p}<0.05,{ }^{* *} \mathrm{p}<0.01,{ }^{* * *} \mathrm{p}<0.001\right)$. siC, silencing control. SIRT6, sirtuin 6; PTC, papillary thyroid cancer.

SIRT6 expression is upregulated in PTC tumor tissues. Microarray data (GSE33630) was used to evaluate SIRT6 gene expression in PTC. A total number of 45 pairs of PTC tissues and their corresponding para-tumor tissues were analyzed. 

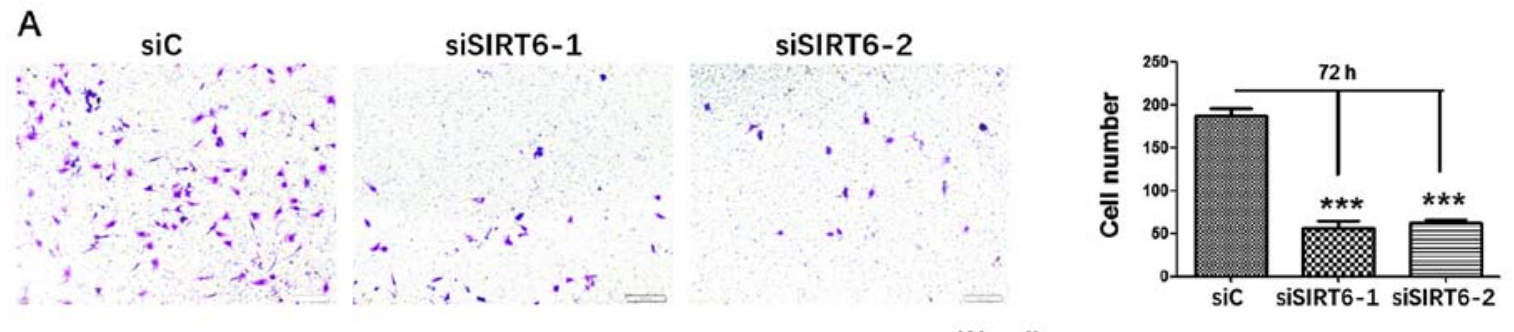

K1 cells
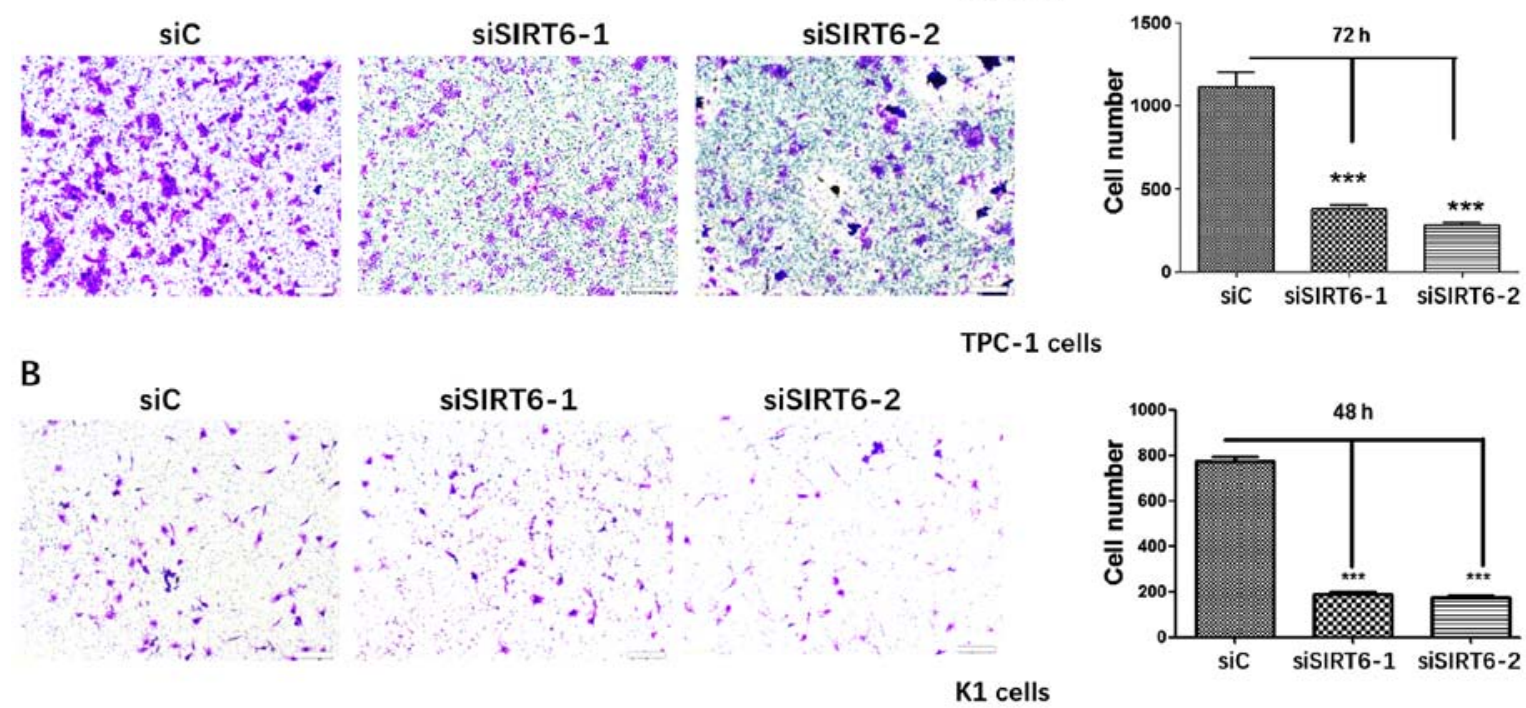

sic

siSIRT6-1

SiSIRT6-2
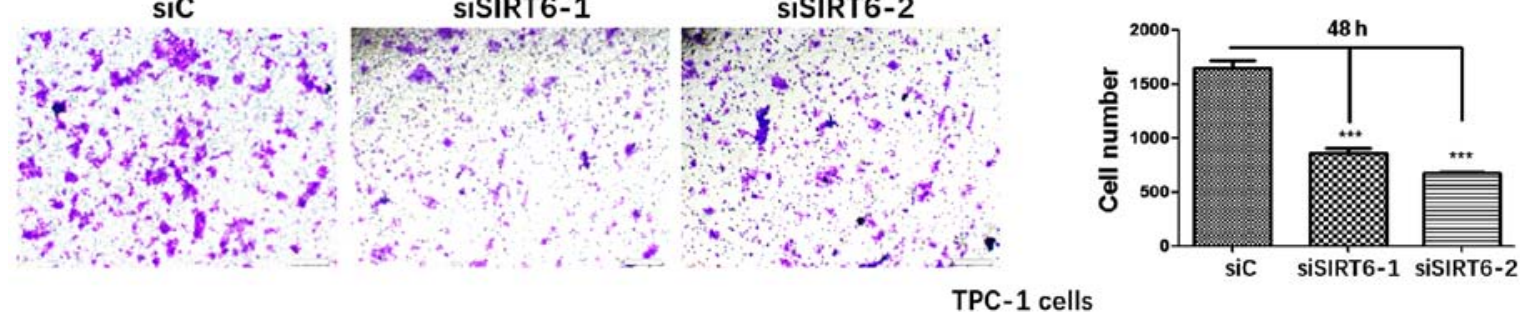

Figure 4. Silencing SITR6 decreased PTC cell migration and invasion in vitro. (A) Migration assays showing knock down of SIRT6 gene resulted in a significant decrease of the migration ability in both K1 and TPC-1 cells. (B) Invasion assays showing silenced SIRT6 inhibited K1 and TPC-1 cell invasion significantly. ( $\left(\mathrm{p}<0.05,{ }^{* * *} \mathrm{p}<0.01,{ }^{* * *} \mathrm{p}<0.001\right)$. siC, silencing control; SIRT6, sirtuin 6; PTC, papillary thyroid cancer.

The expression of SIRT6 was significantly higher in tumor compared to non-tumor tissues ( $\mathrm{p}=0.029$; Fig. $2 \mathrm{~A}$ ). IHC was also adapted to analyze the protein level of SIRT6 expression and subcellular localization. As is shown in Fig. 2B, SIRT6 was expressed in the nuclei of cells and was higher in PTC tumor tissues.

Tissues from 130 patients who were surgically treated and pathologically diagnosed as PTC were obtained to verify SIRT6 expression. The upregulation of SIRT6 was observed in tumor tissues at both mRNA ( $\mathrm{p}=0.011 ; \mathrm{Fig} .2 \mathrm{C})$ and protein levels (Fig. 2D).

Silencing SIRT6 weaken PTC cell proliferation in vitro. To investigate the role of SIRT6 on PTC cell proliferation, we generated SIRT6-silenced TPC-1 and SIRT6-silenced K1 cells. The two shRNAs against SIRT6 decreased gene expression in PTC cells, which was confirmed by qRT-PCR (Fig. 3A) and western blotting (Fig. 3B). Colony formation assay showed that silencing of SIRT6 resulted in significant decrease of cloning capacity of PTC cells in vitro (Fig. 3C).
Cell viability test was then performed by using ATPlite luminescence assay, which displayed that the ATP-lite unit of each experiment group (siSIRT6-1 and siSIRT6-2) was lower than the control group in both cell lines (Fig. 3D). Combined, these results demonstrated that SIRT6 may promote the proliferation in TPC-1 and $\mathrm{K} 1$ cells.

Silencing SIRT6 decreases PTC cell migration and invasion in vitro. Transwell assays were performed on SIRT6-silenced TPC-1 and SIRT6-silenced K1 cells to determine the influence of silenced SIRT6 on PTC cell migration and invasion, respectively. The migration assays showed a significant decrease in the number of migrated cells in both cell lines (Fig. 4A). Similarly, the invasion assays also revealed a huge decrease of cells invaded in K1 and TPC-1 cell (Fig. 4B). These results pointed out that SIRT6 stimulated cell migration and invasion in vitro.

Influence of silencing SIRT6 gene on PTC cell cycle distribution. To further investigate whether silenced SIRT6 alters PTC 
A

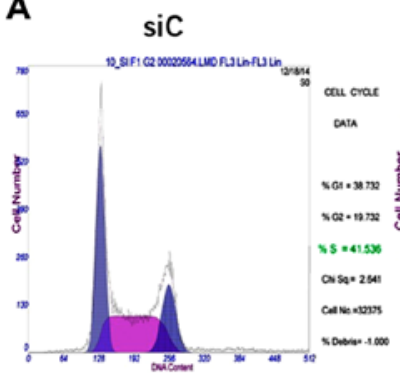

G1\% $38.73 \%$

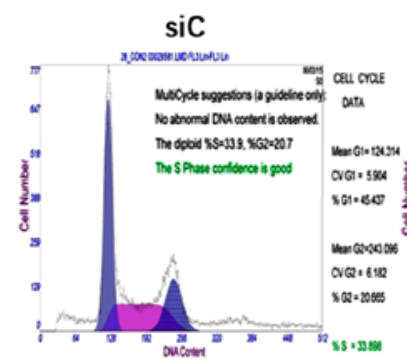

G1\% $\quad 45.44 \%$
siSIRT6-1

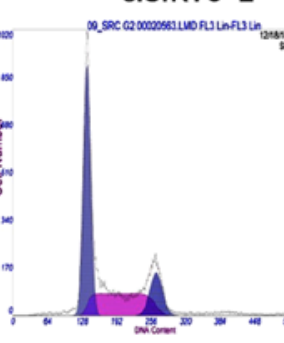

$51.33 \%$
SISIRT6-2

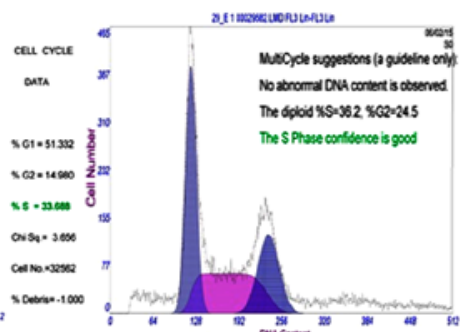

$39.24 \%$
B

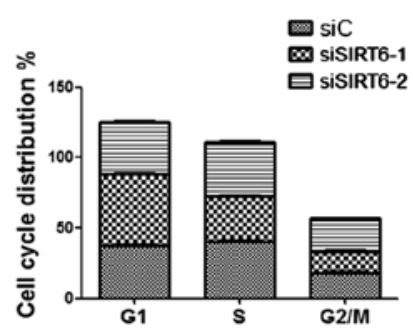

K 1

Figure 5. Effect of SIRT6 silencing on cell cycle distribution of PTC cells. Cell cycle distribution was measured by flow cytometry. (A) Histograms showing cell cycle distribution in control group and two SIRT6-silenced groups of K1 and TPC-1 cell lines. The SIRT6-1 silenced K1 cell had increased proportions of cells in G1 phase. (B) Bar graphs illustrating the proportion of cells in different phases. siC, silencing control; SIRT6, sirtuin 6; PTC, papillary thyroid cancer.

cell cycle distribution, flow cytometry analysis was conducted in TPC-1 and K1 cells. Results unveiled that SIRT6-1 silenced $\mathrm{K} 1$ cells possessed a significant increase in the proportion of cells in G1 phase compared to the control group. However, there was only slightly elevated number of cells in G1 phase of SIRT6-2 silenced K1 cells. As for TPC-1 cells, the proportion of cells in G1 phase decreased in both SIRT6-1 and SIRT6-2 silenced TPC-1 cells (Fig. 5). The inconsistency in the outcome indicated that SIRT6 had a rather complicated role in regulating cell cycle distribution.

SIRT6 enhances PTC cell aggressiveness via BRAF/ERK/Mcl-1 pathway. Western blot analysis was performed to determine if SIRT6 is involved in BRAF/ERK/Mcl-1 signaling pathway. Apoptosis related proteins including NF- $\kappa \mathrm{B}$ and C-PARP were also tested. The protein expression of SIRT6, NF- $\mathrm{B}, \mathrm{C}-\mathrm{PARP}$, phosphorylated ERK and Mcl-1 were analyzed in control and SIRT6-silenced K1 cells, separately. Silencing SIRT6 was accompanied by downregulation of phosphorylated ERK and the apoptosis associated protein Mcl-1, while the expression of $\mathrm{NF}-\kappa \mathrm{B}$ and C-PARP were enhanced (Fig. 6).

\section{Discussion}

SITR6 has been reported in connection with several types of human cancers. Under most of the circumstances, it acts as a tumor suppressor, such as in pancreatic cancer, breast cancer and in hepatocellular carcinomas (14-18). Regulation of aerobic glycolysis in cancer cells and cancer-associated point mutations in SIRT6, may be the foundation of its function as a tumor suppressor $(21,22)$. However recently, some researchers have found SIRT6 can be an oncogene in lung cancer, prostate cancer and skin cancer where it was overexpressed $(19,20,23)$.

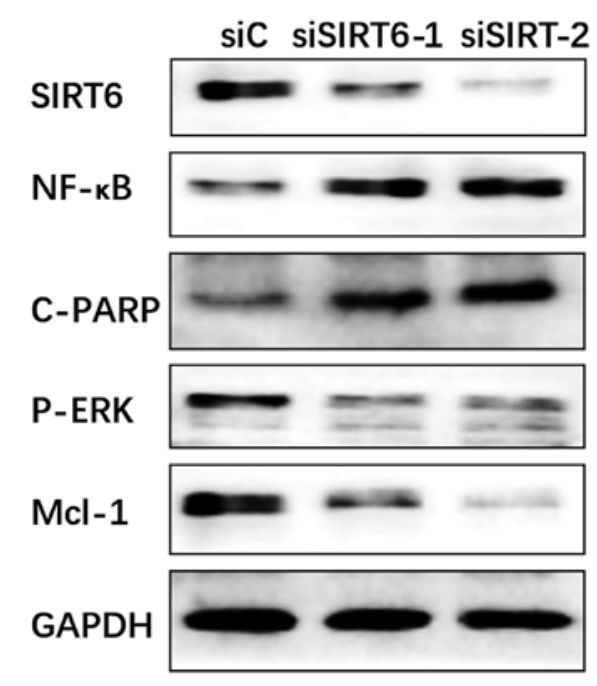

Figure 6. Protein level of SIRT6, NF- $\kappa$ B, C-PARP, phosphorylated ERK and $\mathrm{Mcl}-1$ expression in treated and untreated $\mathrm{K} 1$ cells $\left({ }^{*} \mathrm{p}<0.05,{ }^{* *} \mathrm{p}<0.01\right.$, $\left.{ }^{* * *} \mathrm{p}<0.001\right)$. siC, silencing control; SIRT6, sirtuin 6; PTC, papillary thyroid cancer.

Thus, the function of SIRT6 in cancer is likely context and tissue specific. The underlying mechanisms between SIRT6 and tumor progression is unclear. To our best knowledge, this study is the first to explore the involvement of SIRT6 gene in PTC.

We analyzed SIRTs expression and clinicopathological parameters of the TCGA cohort. Among the 7 SIRTs, SIRT6 was an independent biomarker for nodal metastasis (OR=1.794, 95\% CI: $1.256-1.920, \mathrm{p}=0.012$ ) and related to poor RFS. The X-tile plots further pointed out that when divided by SIRT6 expression volume, PTC prognosis can differ significantly. 
Notably, the higher the expression of SIRT6 in tumor was, the poorer their RFS would be. By microarray analysis, we found SIRT6 was higher in PTC tumor tissues and this result was also confirmed by IHC and the qRT-PCR of our in-house cohort. After successful establishment of SIRT6 silenced K1 and TPC-1 cell lines, we then further demonstrated that the silencing of SIRT6 decreased PTC cell aggressiveness through BRAF/ERK/Mcl-1 pathway in vitro. Taken together, these results may indicate SIRT6 acts as an oncogene in PTC.

Our cell cycle distribution analysis received inconsistent results from SIRT6 silenced K1 and TPC-1 cells. The proportion of cell numbers in G1 phase of SIRT6 silenced K1 cells was significantly increased compared to untreated K1 cells, suggesting SIRT6 may be responsible for cell proliferation. However, no significant increase of cells in G1 phase were observed in the other three cell lines. The $B R A F^{\mathrm{V} 600 \mathrm{E}}$ mutation may be responsible for the disagreement since $\mathrm{K} 1$, instead of TPC-1 cell line is known to harbor $B R A F^{\mathrm{V} 600 \mathrm{E}}$ mutation. Activating BRAF mutation is frequent, being the most common oncogenic mutation in PTC (24). Also, the presence of the $B R A F^{\mathrm{V} 600 \mathrm{E}}$ mutation was acknowledged to be associated with poor prognosis among patients with PTC $(6,25)$. It can be inferred that SIRT6 plays a quite complicated role in controlling PTC cell cycle distribution, which concerns BRAF.

We have demonstrated that silencing SIRT6 was accompanied by a downregulation of Mcl-1 and phosphorylated ERK, while NF- $\kappa \mathrm{B}$ and C-PARP were elevated. On these grounds, we speculate that SIRT6 is involved in the BRAF/ERK/Mcl-1 pathway. BRAF promotes the stabilizing phosphorylation of the anti-apoptotic protein Mcl-1, which was frequently reported in melanoma and colorectal cancer $(26,27)$. ERK is known to be a member of the mitogen-activated protein kinase (MAPK) signaling pathway and is markedly increased in PTC cells driven by oncogenic BRAF. The ERK pathway suppresses cellular senescence by inhibiting the expression of p16 and p21, thus it is crucial in sustaining cell proliferation and activating tumorigenesis (28). ERK was also found to mediate Mcl-1 stabilization in colon cancer and hepatic cancers, which may be another essential factor in cancer cell survival (29,30). Activation of ERK and Mcl-1 stabilization is $B R A F^{\mathrm{V} 600 \mathrm{E}}$-dependent and related to the disruption of S6K1-IRS-2/PI3K negative feedback loop (29). Bai et al presented that SIRT6 overexpression increased ERK activation via ERK1/2/MMP9 axis in non-small cell lung cancer (23). In hepatocellular carcinoma, transforming growth factor- $\beta 1 / \mathrm{H} 2 \mathrm{O} 2 / \mathrm{HOCl}$ could upregulate SIRT6 expression by inducing the sustained activation of ERK (31). Anti-apoptosis related proteins such as NF- $\kappa \mathrm{B}$ and c-PAPP which is a fragment of caspase-3 cleaved PARP that exists during apoptosis were analyzed. Previous studies also showed that SIRT6 attenuates $\mathrm{NF}-\kappa \mathrm{B}$ signaling via $\mathrm{H} 3 \mathrm{~K} 9$ deacetylation at chromatin (32). $\mathrm{NF}-\kappa \mathrm{B}$ levels were significantly and negatively correlated with SIRT6 mRNA levels in myocardial hypoxia/reoxygenation induced injury and other diseases $(12,33)$. Despite the above, SIRT6 was found to directly inhibit the expression of a subset of NF- $\mathrm{B}$ target genes, especially those associated with senescence (32). This implies that SIRT6 have the potential to promote tumor development considering its negative effect on cellular senescence and that NF- $\kappa \mathrm{B}$ is an important anti-apoptotic factor. Combined, SIRT6 may be involved in the
BRAF/ERK/Mcl-1 axis, by which it promotes cell aggressiveness in PTC.

In conclusion, our study demonstrated that SIRT6 upregulation was associated with poor RFS in PTC patients and may promote PTC development and progression. Furthermore, we demonstrated that SIRT6 promoted tumor progression through the pathway. SIRT6 may serve as a potential therapeutic target in PTC and its utility as a prognostic indicator warrants further study.

\section{Acknowledgements}

This study was supported by funds from the National Science Foundation of China (nos. 81572622 and 81272934 to QHJ).

\section{References}

1. Davies L and Welch HG: Current thyroid cancer trends in the United States. JAMA Otolaryngol Head Neck Surg 140: 317-322, 2014.

2. Colonna M, Uhry Z, Guizard AV, Delafosse P, Schvartz C, Belot A and Grosclaude P; FRANCIM network: Recent trends in incidence, geographical distribution, and survival of papillary thyroid cancer in France. Cancer Epidemiol 39: 511-518, 2015.

3. Siegel RL, Miller KD and Jemal A: Cancer Statistics, 2017. CA Cancer J Clin 67: 7-30, 2017.

4. McLeod DS, Sawka AM and Cooper DS: Controversies in primary treatment of low-risk papillary thyroid cancer. Lancet 381: 1046-1057, 2013.

5. La Vecchia C, Malvezzi M, Bosetti C, Garavello W, Bertuccio P, Levi F and Negri E: Thyroid cancer mortality and incidence: A global overview. Int J Cancer 136: 2187-2195, 2015.

6. Xing M, Alzahrani AS, Carson KA, Shong YK, Kim TY, Viola D, Elisei R, Bendlová B, Yip L, Mian C, et al: Association between BRAF V600E mutation and recurrence of papillary thyroid cancer. J Clin Oncol 33: 42-50, 2015.

7. Grogan RH, Kaplan SP, Cao H, Weiss RE, Degroot LJ, Simon CA, Embia OM, Angelos P, Kaplan EL and Schechter RB: A study of recurrence and death from papillary thyroid cancer with 27 years of median follow-up. Surgery 154: 1436-1446, discussion 1446-1447, 2013.

8. Bartosch C, Monteiro-Reis S, Almeida-Rios D, Vieira R, Castro A, Moutinho M, Rodrigues M, Graça I, Lopes JM and Jerónimo C: Assessing sirtuin expression in endometrial carcinoma and non-neoplastic endometrium. Oncotarget 7: 1144-1154, 2016.

9. Mouchiroud L, Houtkooper RH, Moullan N, Katsyuba E, Ryu D, Cantó C, Mottis A, Jo YS, Viswanathan M, Schoonjans K, et al: The NAD(+)/Sirtuin pathway modulates longevity through activation of mitochondrial UPR and FOXO signaling. Cell 154: 430-441, 2013.

10. Lai CC, Lin PM, Lin SF, Hsu CH, Lin HC, Hu ML, Hsu CM and Yang MY: Altered expression of SIRT gene family in head and neck squamous cell carcinoma. Tumour Biol 34: 1847-1854, 2013.

11. Zhang $X$, Khan S, Jiang H, Antonyak MA, Chen X, Spiegelman NA, Shrimp JH, Cerione RA and Lin H: Identifying the functional contribution of the defatty-acylase activity of SIRT6. Nat Chem Biol 12: 614-620, 2016.

12. Toiber D, Erdel F, Bouazoune K, Silberman DM, Zhong L, Mulligan P, Sebastian C, Cosentino C, Martinez-Pastor B, Giacosa S, et al: SIRT6 recruits SNF2H to DNA break sites, preventing genomic instability through chromatin remodeling. Mol Cell 51: 454-468, 2013.

13. Wang WW, Zeng Y, Wu B, Deiters A and Liu WR: A chemical biology approach to reveal Sirt6-targeted Histone H3 sites in nucleosomes. ACS Chem Biol 11: 1973-1981, 2016.

14. Kugel S, Sebastián C, Fitamant J, Ross KN, Saha SK, Jain E, Gladden A, Arora KS, Kato Y, Rivera MN, et al: SIRT6 Suppresses Pancreatic Cancer through Control of Lin28b. Cell 165: 1401-1415, 2016.

15. Marquardt JU, Fischer K, Baus K, Kashyap A, Ma S, Krupp M, Linke M, Teufel A, Zechner U, Strand D, et al: Sirtuin-6-dependent genetic and epigenetic alterations are associated with poor clinical outcome in hepatocellular carcinoma patients. Hepatology 58: 1054-1064, 2013. 
16. Elhanati S, Ben-Hamo R, Kanfi Y, Varvak A, Glazz R, Lerrer B, Efroni S and Cohen HY: Reciprocal regulation between SIRT6 and miR-122 controls liver metabolism and predicts hepatocarcinoma prognosis. Cell Rep 14: 234-242, 2016.

17. Thirumurthi U, Shen J, Xia W, LaBaff AM, Wei Y, Li CW, Chang WC, Chen CH, Lin HK, Yu D, et al: MDM2-mediated degradation of SIRT6 phosphorylated by AKT1 promotes tumorigenesis and trastuzumab resistance in breast cancer. Sci Signal 7: ra71, 2014

18. Khongkow M,Olmos Y,Gong C,Gomes AR,MonteiroLJ, YagüeE, Cavaco TB, Khongkow P, Man EP, Laohasinnarong S, et al: SIRT6 modulates paclitaxel and epirubicin resistance and survival in breast cancer. Carcinogenesis 34: 1476-1486, 2013.

19. Ming M, Han W, Zhao B, Sundaresan NR, Deng CX, Gupta MP and He YY: SIRT6 promotes COX-2 expression and acts as an oncogene in skin cancer. Cancer Res 74: 5925-5933, 2014.

20. Xie Q, Wong AS and Xia W: SIRT6 induces EMT and promotes cancer cell invasion and migration in prostate cancer. In: Proceedings of the 105th Annual Meeting of the American Association for Cancer Research. Cancer Res 74 (Suppl 19): Abst $1151,2014$.

21. Kugel S, Feldman JL, Klein MA, Silberman DM, Sebastián C, Mermel C, Dobersch S, Clark AR, Getz G, Denu JM, et al: Identification of and molecular basis for SIRT6 loss-of-lunction point mutations in cancer. Cell Rep 13: 479-488, 2015.

22. Sebastián C, Zwaans BM, Silberman DM, Gymrek M, Goren A, Zhong L, Ram O, Truelove J, Guimaraes AR, Toiber D, et al: The histone deacetylase SIRT6 is a tumor suppressor that controls cancer metabolism. Cell 151: 1185-1199, 2012.

23. Bai L, Lin G, Sun L, Liu Y, Huang X, Cao C, Guo Y and Xie C: Upregulation of SIRT6 predicts poor prognosis and promotes metastasis of non-small cell lung cancer via the ERK1/2/MMP9 pathway. Oncotarget 7: 40377-40386, 2016.

24. Liu X, Qu S, Liu R, Sheng C, Shi X, Zhu G, Murugan AK, Guan $\mathrm{H}, \mathrm{Yu} \mathrm{H}$, Wang Y, et al: TERT promoter mutations and their association with BRAF V600E mutation and aggressive clinicopathological characteristics of thyroid cancer. J Clin Endocrinol Metab 99: E1130-E1136, 2014.

25. Howell GM, Nikiforova MN, Carty SE, Armstrong MJ, Hodak SP, Stang MT, McCoy KL, Nikiforov YE and Yip L: BRAF V600E mutation independently predicts central compartment lymph node metastasis in patients with papillary thyroid cancer. Ann Surg Oncol 20: 47-52, 2013.
26. Becker TM, Boyd SC, Mijatov B, Gowrishankar K, Snoyman S, Pupo GM, Scolyer RA, Mann GJ, Kefford RF, Zhang XD, et al: Mutant B-RAF-Mcl-1 survival signaling depends on the STAT3 transcription factor. Oncogene 33: 1158-1166, 2014.

27. Kawakami H, Huang S, Pal K, Dutta SK, Mukhopadhyay D and Sinicrope FA: Mutant BRAF upregulates MCL-1 to confer apoptosis resistance that is reversed by MCL-1 antagonism and cobimetinib in colorectal cancer. Mol Cancer Ther 15: 3015-3027, 2016.

28. Liu D, Liu J, Lin B, Liu S, Hou R, Hao Y, Liu Q, Zhang S and Iwamori M: Lewis y regulate cell cycle related factors in ovarian carcinoma cell RMG-I in vitro via ERK and Akt signaling pathways. Int J Mol Sci 13: 828-839, 2012.

29. He K, Chen D, Ruan H, Li X, Tong J, Xu X, Zhang L and Yu J: BRAFV600E-dependent Mcl-1 stabilization leads to everolimus resistance in colon cancer cells. Oncotarget 7: 47699-47710, 2016.

30. Gao M, Kong Q, Hua H, Yin Y, Wang J, Luo T and Jiang Y: AMPK-mediated up-regulation of mTORC2 and MCL-1 compromises the anti-cancer effects of aspirin. Oncotarget 7: 16349-16361, 2016

31. Feng XX, Luo J, Liu M, Yan W, Zhou ZZ, Xia YJ, Tu W, Li PY, Feng ZH and Tian DA: Sirtuin 6 promotes transforming growth factor- $\beta 1 / \mathrm{H} 2 \mathrm{O} 2 / \mathrm{HOCl}$-mediated enhancement of hepatocellular carcinoma cell tumorigenicity by suppressing cellular senescence. Cancer Sci 106: 559-566, 2015.

32. Kawahara TL, Michishita E, Adler AS, Damian M, Berber E, Lin M, McCord RA, Ongaigui KC, Boxer LD, Chang HY, et al: SIRT6 links histone H3 lysine 9 deacetylation to NF-kappaB-dependent gene expression and organismal life span. Cell 136: 62-74, 2009.

33. Cheng MY, Cheng YW, Yan J, Hu XQ, Zhang H, Wang ZR, Yin Q and Cheng W: SIRT6 suppresses mitochondrial defects and cell death via the NF- $\mathrm{B}$ pathway in myocardial hypoxia/reoxygenation induced injury. Am J Transl Res 8: 5005-5015, 2016. 